\title{
Medical Applications for Implantable Devices Utilizing Electromagnetic Fields
}

\author{
Hidetoshi Matsuki \\ Professor \\ Department of Electrical and Communication Engineering, Graduate School of Engineering \\ E-mail: matsuki@ecei.tohoku.ac.jp
}

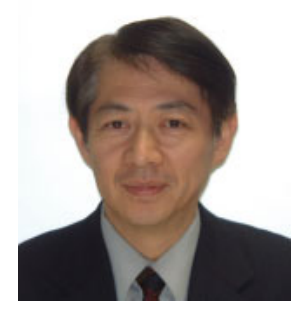

\begin{abstract}
We approached various researches, which contain developments of functional hyperthermia, thermotherapy by using metallic stent, implanted FES system, transcutaneous power-signal supply system for implantable total artificial heart, rechargeable power supply system for a cardiac pacemaker, and temperature control for SMA artificial anal sphincter. We are examining the in vivo application of electromagnetic fields with regard to biomagnetism and energy management. From the perspective of electrical engineering, the power source of equipment is an important issue for the majority of medical devices. This paper shows the progress of the functional hyperthermia system in our researches.
\end{abstract}

\section{Introduction}

We are examining the in vivo application of electromagnetic fields with regard to biomagnetism and energy management. From the perspective of electrical engineering, the power source of equipment is an important issue for the majority of medical devices. Power savings (i.e., low power consumption) and the use of wireless equipment hold the key to promoting the widespread use of medical equipment in a variety or areas, including social welfare and nursing. The next generation of electrical equipment should not need to be connected directly to a power source. Contactless power transfer will benefit medical equipment. When such equipment can be mounted to a device with no internal energy source, a dramatic improvement of the health system is envisioned. We expect such equipment to contribute greatly to medical treatment in the 21 st Century. Medical equipment that constitues a fully self-contained system with noncontact energy transmission includes the following:

Type A: Uses transferred energy to power implaned devices, such as artificial hearts and micromachines;

Type B: Uses electrical power, such as in Functional Electrical Stimulation Systems; and

Type C: Uses a heat source, such as in devices that produce local hyperthermia.

From the viewpoints, We approached various researches, which contain developments of functional hyperthermia system, thermotherapy by using metallic stent, implanted FES system, transcutaneous powersignal supply system for implantable total artificial heart, rechargeable power supply system for a cardiac pacemaker, and temperature control for SMA artificial anal sphincter.

This paper shows the progress of the functional hyperthermia system in our researches.

\section{Functional Hyperthermia 2.1. Hyperthermia thermotherapy}

Various forms of cancer therapy, including chemotherapy, radiotherapy, hyperthermia and immunotherapy, have been developed. Among them, hyperthermia is known as a therapeutic technique that raises body temperature to destroy tumors. Because of the applications of physical energy from outside the body, hyperthermia is a minimally invasive treatment, and thus expectations for this therapy are high.

Hyperthermia induces tumor necrosis by the use of heat, and it is generally well known that at temperatures in excess of $42.5{ }^{\circ} \mathrm{C}$, the survival rate of tumor tissues drops sharply. In addition, the temperature of tumor tissues easily rises compared to that of normal cells, due to a higher heat sensitivity and a smaller cooling effect of blood flow, which then leads to advantages in using the hyperthermia.

On the other hand, hyperthermia has been faced with difficulties in the temperature control and localized heating of tissue. In order to improve performance, implant heating system to embed heat elements have come under review. As one of the methods of the implant type, we have proposed "Soft-Heating Method" [1,2].

When elements are embedded under the implant heating system, the temperature may be different between the central and marginal parts. To eliminate the difference, it is desirable to ensure temperature uniformity at or above $42.5{ }^{\circ} \mathrm{C}$ including that of the marginal part, by raising the temperature higher than the lethal temperature for the tumor. For destruction of tumor tissues, we have proposed the application of high-temperature hyperthermia, as shown Fig.1.

Although it is necessary to implant the heat element in the tumor tissue, there are various advantages on hyperthermia, such as preventing bacterial infection 


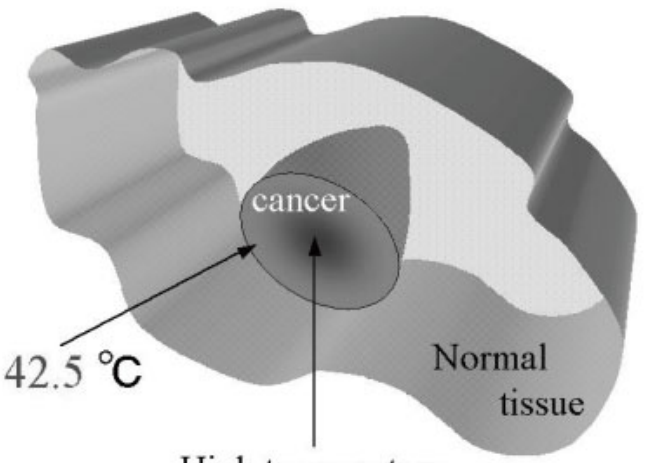

High temperature

Fig. 1. Conception of High-temperature Hyperthermia.

and allowing easy retreatment as well as localized heating of tissue. This is because the method enables non-contact heating from outside the body.

\subsection{Heat generation mechanism 2.2.1. Soft-heating method}

The soft-heating method is an inductive heating process that uses a thermosensitive magnetic material as an embedded heat element. In this method, the element produces heat in high-frequency alternating magnetic field, and has features of automatic temperature control utilizing Curie point of a thermosensitive magnetic material. The method is suitable for localized heating in tumor tissue located in deep parts of the body.

In this study, the soft-heating method is applied for the purpose of achieving high-temperature Hyperthermia. We made the functional hyperthermia which has the heat element is composed of a metal ring, and a thermosensitive magnetic material. The thermosensitive magnetic material is employed for the effect of magnetic flux density and the temperature control of the heat element. Similarly, the metal ring is wrapped around the thermosensitive magnetic material in order to generate a larger quantity of heat than a single piece of magnetic material.

\subsubsection{Heating mechanism}

Figure 2 displays the principle of operation of heat elements (functional hyperthermia). When the temperature of the thermosensitive magnetic material is lower than the Curie point $\left(\mathrm{T}_{\mathrm{C}}\right)$, the magnetic flux is concentrated by the effect of high permeability of the magnetic material. The short-circuit current is then induced in the copper ring to raise the temperature of

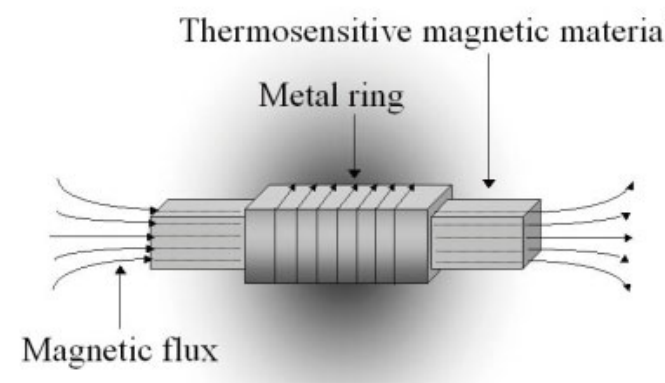

(a) Temperature of magnetic material is lower than Curie point.

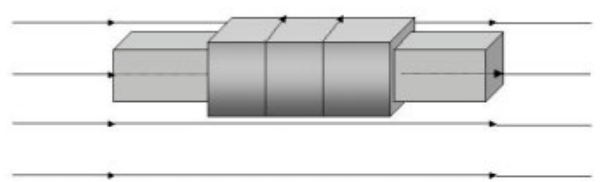

(b) Temperature of magnetic material is higher than Curie point.

Fig. 2. Heating mechanism of the element.

the heat element. On the other hand, when the temperature of the thermosensitive agnetic material is higher than the Curie point, the density of the magnetic flux disappears, thus resulting in loss of heat generation.

Since the temperature of the heat element does not rise more than that of the Curie point, temperature control is achieved with the use of a Curie point as criteria $[3,4]$.

\section{Heat Characteristics of Micro Magnetic Heat Elements for Functional Hyperthermia [5] 3.1. Experiment}

In this chapter, the authors examined the heat characteristics of heat elements with various volumes to adjust to the size of each tumor tissue. Further improvement will require more compact design to ease the burden of patients. The study aims to clarify the temperature characteristics of heat elements with various volumes.

The thermosensitive magnetic material used for experiments is $\mathrm{Ni}-\mathrm{Cu}-\mathrm{Zn}$ ferrite (relative permea-bility: 2100, saturation magnetization: $320 \mathrm{mT}$ ) and the Curie point is $90{ }^{\circ} \mathrm{C}$. As a metal ring, the copper ring is selected, and the ring of $0.15 \mathrm{~mm}$ in thickness has been wrapped around each thermosensitive magnetic material. For this study, volume change is measured by changing the length and the cross-sectional area of the magnetic material.

The experiments provide two sets of ten sample magnetic materials: one set for 2, 4, 6, 8, and $10 \mathrm{~mm}$ in length, with a constant cross-sectional area of $10 \mathrm{~mm}^{2}$, 
and one for $1.6,3.6,4.9,6.4$, and $10 \mathrm{~mm}^{2}$ in crosssectional area, with a constant length of $10 \mathrm{~mm}$.

An experimental heat element, which is placed in the insulator, is thermally excited through the use of a solenoidal coil, as shown in Fig. 3 . The coil specification is $8 \mathrm{mT}$ at $100 \mathrm{kHz}$. The thermometry device used is a fluorescent thermometer that is hardly affected by a high-frequency magnetic field.

Head of thermometer probe was bonding at the center part of metal ring.

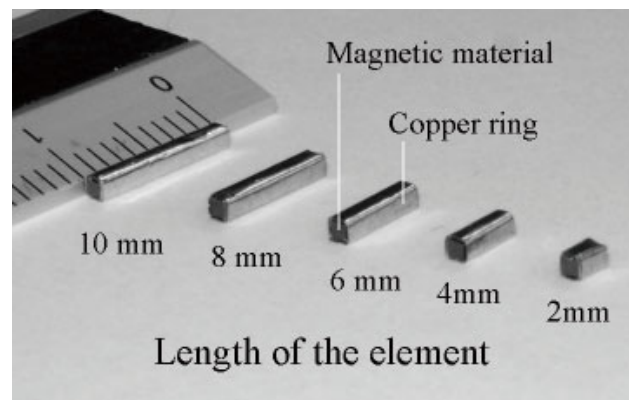

(a) Outline of the heat element (The cross-sectional area of ferrite: $1 \mathrm{~mm}^{2}$, Thickness of the copper ring: $0.15 \mathrm{~mm})$.

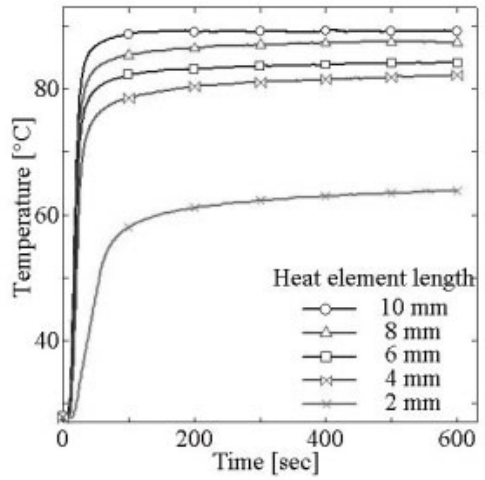

(b) Temperature when the length of the heat element was changed.

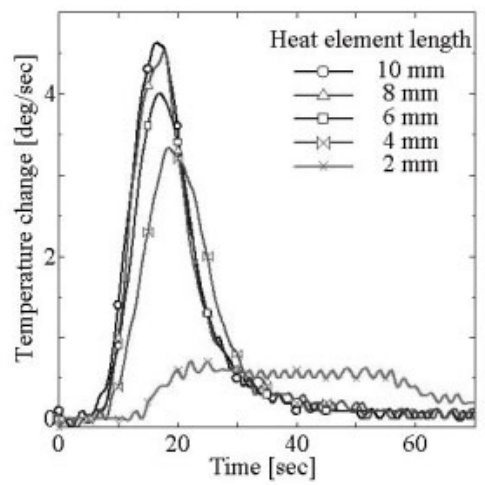

(c) Initial temperature change when the length of the heat element was changed.

Fig. 4. Temperature characteristics of the element with different length.

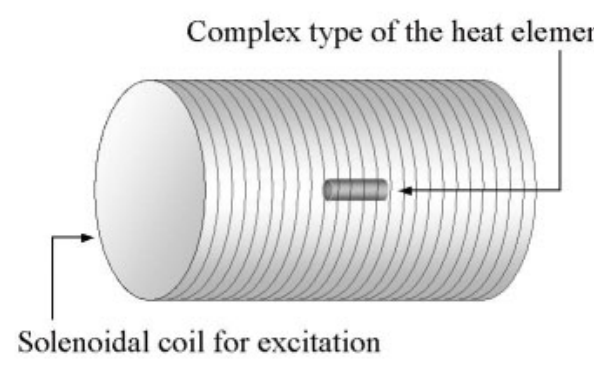

Fig. 3. Construction of excitation system. (The magnetic field of $8 \mathrm{mT}$ has been generated in the central part of solenoidal coil.)

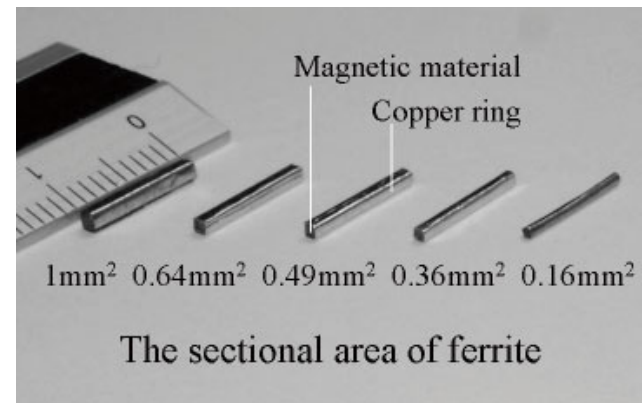

(a) Outline of the heat element (Length of the ferrite: $10 \mathrm{~mm}$, Thickness of the copper ring: $0.15 \mathrm{~mm}$ ).

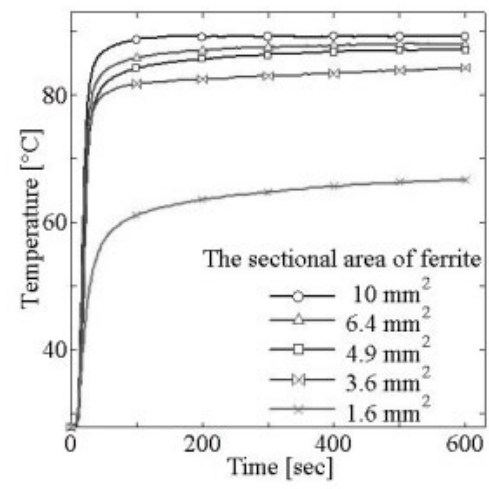

(b) Temperature when the cross-sectional area of the heat element was changed.

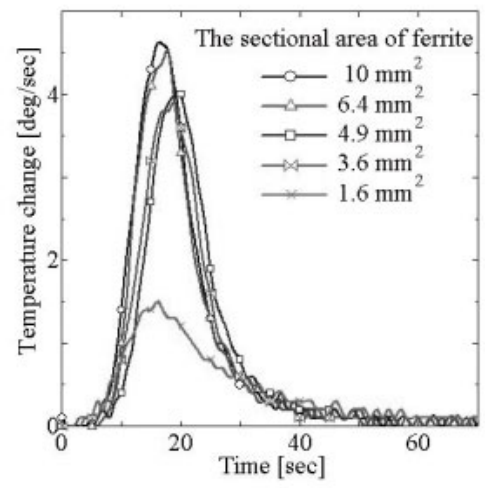

(c) Initial temperature change when the crosssectional area of ferrite was changed.

Fig. 5. Temperature characteristics of the element with different cross-sectional area. 


\subsection{Result}

Outline of the heat element displayed in the Fig. 4-a and Fig. 5-a. Figure 4-b demonstrates the temperature characteristics when the lengths of the heat elements are changed, while Fig. 4-c shows the initial temperature rise.

The temperature measurement point is located at the central part of each metal ring surface.

As the temperature characteristics, the following features were observed: The excitation caused an immediate, rapid temperature rise followed by an end of the rise, then the steady state characteristics were maintained. Consequently, the study found that the attainment temperature of the heat element rose with increasing element volume. In this experimental condition, where the length of the heat element was increased up to $10 \mathrm{~mm}$, temperature has been controlled at the set temperature (Curie point).

Additionally, the temperature characteristics show the saturation tendency as the element volume increases, though elements in more volume have higher properties. It is also seen that there is a remarkably rapid change of the temperature characteristics in lengths of the heat elements between $2 \mathrm{~mm}$ and $4 \mathrm{~mm}$.

Figure 5-b displays the temperature characteristics when the cross-sectional area of the heat element is changed, while Fig. 5-c shows the initial temperature rise. As with the change in the length, an increase of the cross-sectional area leads to a saturation tendency.

It is also observed that there is a remarkably rapid change of the temperature characteristics in crosssectional area of the heat elements between $1.6 \mathrm{~mm}^{2}$ and $3.6 \mathrm{~mm}^{2}$. A difference in amount of magnetic flux which concentrates on magnetic materials per element, is considered as a factor influencing the temperature characteristics change. Since the amount of magnetic flux is presumably proportional to the effective permeability accompanied by a change of the crosssectional area of the linkage and the volume in the magnetic field, it is thought that this difference causes a change in the short-circuit current induced in the copper ring.

\subsection{Conclusion}

In this chapter, we examined temperature characteristics of heat elements with various volumes to adjust to the size of each tumor tissue. The results provided evidence that the temperature characteristics show a saturation tendency with an increase of the element volume. Based on this tendency, it seems reasonable to suggest that sufficient heating can be obtained, even on smaller element volumes.
Therefore, when considering heating per unit volume, it is expected that a volume with the most effective heating temperature can be obtained.

\section{Distribution of In-Vitro Temperature Rise [6] 4.1. Purpose and outline}

Heaters to be implanted are expected for small size. If we can realize the temperature distribution corresponding to the shape of the tumor, we can treat it appropriately. In this investigation, we examined the effect of wrapped metal ring around thermosensitive magnetic material and basic temperature rise characteristic using one hybrid heater. And we measured $42.5{ }^{\circ} \mathrm{C}$ or more effective area that becomes a tumor necrosis reference temperature when two heaters were used.

\subsection{Experimental condition}

The exciting coil is set up in the thermostat kept 37 ${ }^{\circ} \mathrm{C}$. The specification of this coil is $6.2 \mathrm{mT}$ at $101 \mathrm{kHz}$. The thermometry device we used is a fluorescent thermometer that does not be affected by a highfrequency magnetic field. The thermosensitive magnetic material is Ni-Cu- $\mathrm{Zn}$ ferrite whose Curiepoint is $110^{\circ} \mathrm{C}$ and whose size is $1 \mathrm{~mm} \times 1 \mathrm{~mm} \times 10$ $\mathrm{mm}$. As a metal ring, we wrapped copper tape $(10 \mathrm{~mm}$ in width and $35 \mu \mathrm{m}$ in thickness) around the ferrite and turned it five turns. As a phantom model, $5 \%$ agar was used whose shape was columnar and size was $30 \mathrm{~mm}$ in diameter and $100 \mathrm{~mm}$ in length. The probe of the thermometer was a needle, and it was inserted into the agar phantom, beside the hybrid heater. We measured the temperature rise process of each heater in the phantom model.

\subsection{Comparison among thermosensitive magnetic material, metal Ring, and hybrid Heater}

To confirm the operation of the hybrid heater, we measured the temperature rise characteristic with thermosensitive magnetic material, metal ring, and hybrid heater. The thermometry point is a center part of each element. Fig. 6 shows the measurement result. We can confirm that the temperature of hybrid heater is raised and that of metal ring or ferrite is not changed. That is, it is sure that the eddy current loss in copper ring depends on the flux as a function of temperature; hysteresis loss in ferrite is neglected. Therefore, a hybrid heater is available as a heating unit. 


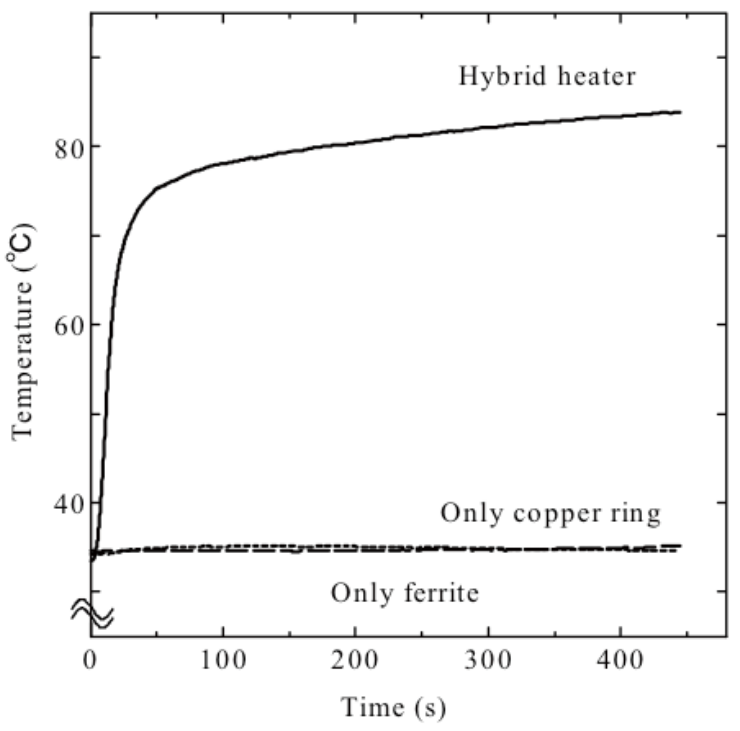

Fig. 6. Measure temperature vs. time for ferrite, copper ring, and hybrid heater.

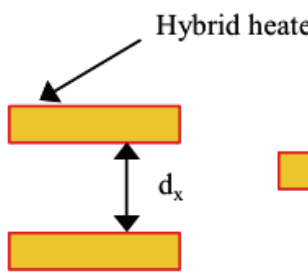

Pattern A

Direction of magnetic flux

Fig. 7. Arrangement of two hybrid heaters.

\subsection{Using two heaters}

To achieve the expansion of $42.5{ }^{\circ} \mathrm{C}$ or more effective area, we measured the area by using two heaters arranged two patterns as shown in Fig. 7, changing the value of $\mathrm{dx}((\mathrm{a}): 10 \mathrm{~mm},(\mathrm{~b}): 20 \mathrm{~mm})$ and dy ((c): $0 \mathrm{~mm},(\mathrm{~d}): 10 \mathrm{~mm})$. Figure 8 shows the represents by two heaters. From the result, we can see measurement result. The white color ovals represent an effective area of one heater, and the gray ones the appearance to which the $42.5^{\circ} \mathrm{C}$ or more effective area has been expanded by overlapping heat from each heater. As a result, it was understood that the temperature distribution modality of the arbitrary shape

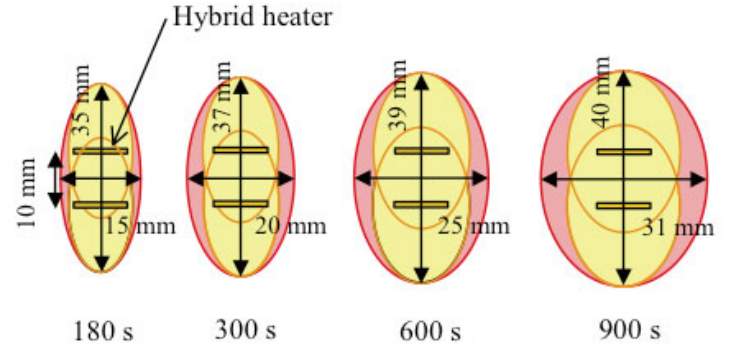

(a) $\mathrm{d}_{\mathrm{x}}=10 \mathrm{~mm}$

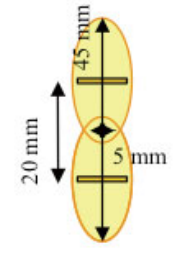

$180 \mathrm{~s}$

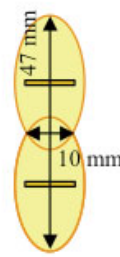

$300 \mathrm{~s}$

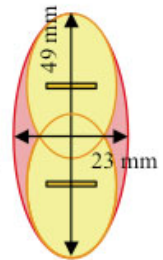

$600 \mathrm{~s}$

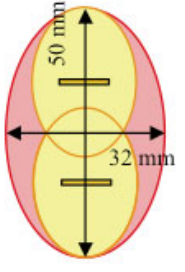

$900 \mathrm{~s}$ (b) $\mathrm{d}_{\mathrm{x}}=20 \mathrm{~mm}$

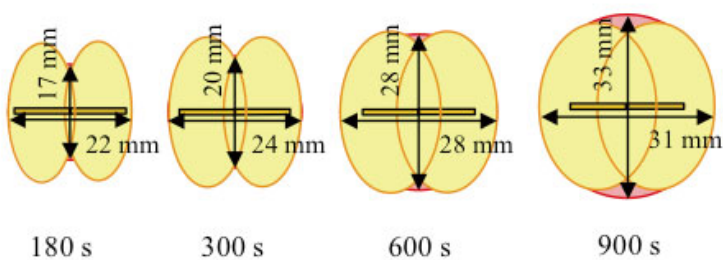

(c) $\mathrm{d}_{\mathrm{y}}=0 \mathrm{~mm}$

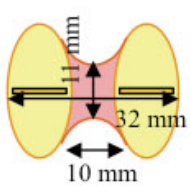

$180 \mathrm{~s}$

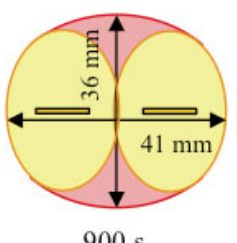

900 s

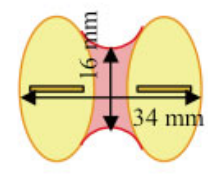

$300 \mathrm{~s}$

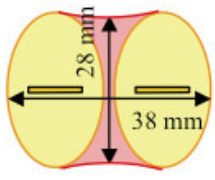

$600 \mathrm{~s}$
Fig. 8. Effective area of $42.5{ }^{\circ} \mathrm{C}$ or more in using two hybrid heaters when the values of $d_{x}$ and $d_{y}$ are changed. 
could be made by devising how to arrange heaters according to the size and shape of tumor.

\subsection{Conclusion}

As a heating method of the hyperthermia, using soft heating method with hybrid heaters, we examined the expansion of effective area. As a result, it is confirmed that the standard of $42.5{ }^{\circ} \mathrm{C}$ or more effective area where tumor could be necrotized. It is assumed that no surgery such as insertion with injector etc., so it is essential to make the heater more small size and high performance. And we are now examining the transportation of the heater by magnetic micromachine that can drive in vivo [4], [7].

\section{Exciting Condition for the High Temperature Magnetic Hyperthermia [8] 5.1. Purpose and outline}

In this investigation, the heater is required to efficiently generate high power. To achieve this proposition, the exciting frequency and magnetic flux density should be changed. In this chapter, we examined the effect of the exciting frequency and magnetic flux density on the heating value of the complex type of heater. And examined the condition which can heat actual tumor.

\subsection{Experimental conditions}

The thermosensitive ferrite used for experiments is $\mathrm{Ni}$ - $\mathrm{Cu}-\mathrm{Zn}$ ferrite $(1 \times 1 \times 10 \mathrm{~mm}$, relative permeability: 2100, saturation magnetization: $320 \mathrm{mT}$ ) and the Curie-point is $90{ }^{\circ} \mathrm{C}$. This point is lower than the boiling point of water. If the Curie-point is selected near $42.5{ }^{\circ} \mathrm{C}$, which is the temperature of the tumor necrosis, the temperature of the tumor area tangent to the heater rises to $42.5^{\circ} \mathrm{C}$, though the temperature of marginal area of the tumor does not rise to the point.

So, it is necessary to use the higher Curie-point than $42.5{ }^{\circ} \mathrm{C}$ and attempt localized heating of the tumor. As a metal ring, the copper ring $(0.05 \mathrm{~mm}$ in thickness $)$ is selected. Fig. 9 is the picture of the heater. An experimental heater, which is placed in the insulator, is thermally excited through the use of a solenoidal coil.

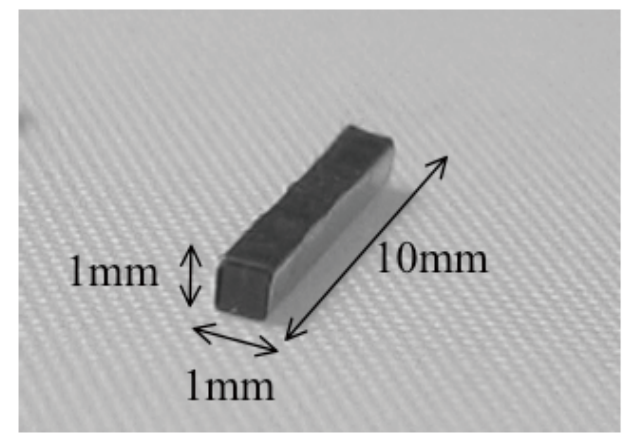

Fig. 9. Picture of the heater.

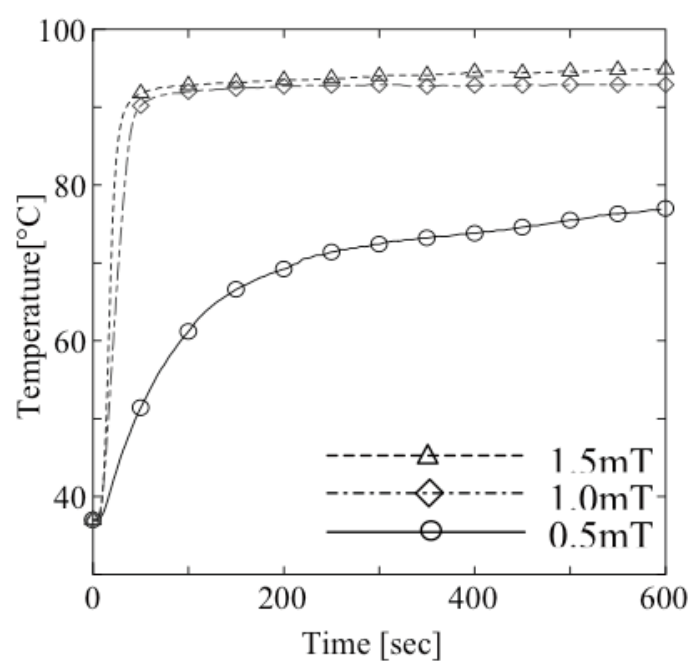

Fig. 11. Temperature characteristics when magnetic flux density is changed.
Fig. 10. Temperature characteristics when exciting frequency is changed. 


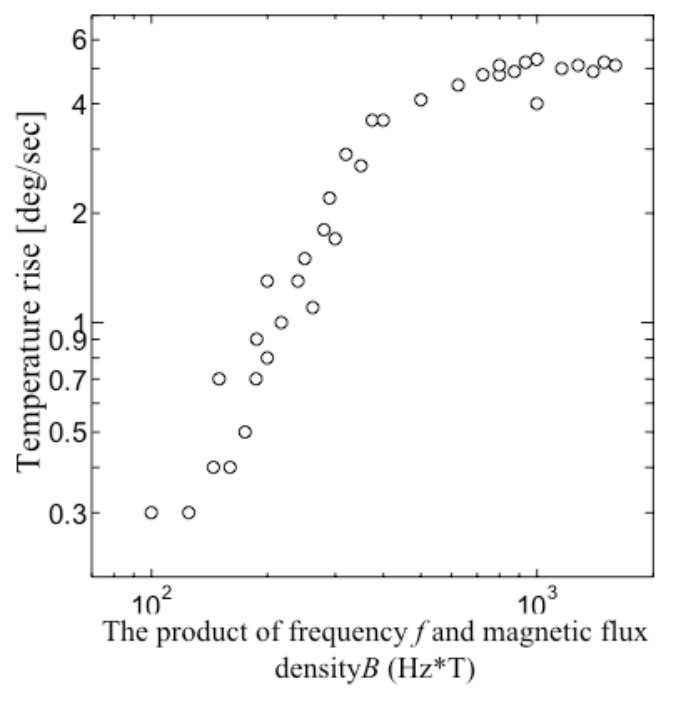

Fig. 12. The temperature rise of the heater as a function of the product of Frequency $f$ and magnetic flux density $B$.

\subsection{Examination of exciting conditions 5.3.1. Temperature characteristics}

Figure 10 shows the temperature characteristics when exciting frequency is changed, while Fig. 11 shows the temperature characteristics when magnetic flux density is changed. It is observed that the heating value of the heater increases as exciting frequency rises or magnetic flux density increases.

\subsubsection{Temperature rise and highest attainment temperature}

The short - circuit current in the metal ring is the function of $f^{*} B$. Figure 12 shows the temperature rise of the heater as a function of the product of frequency $f$ and magnetic flux density $\mathrm{B} ; \mathrm{f} * \mathrm{~B}\left(\mathrm{~Hz}^{*} \mathrm{~T}\right)$. Figure 13 shows the highest attainment temperature as a function of $\mathrm{f}^{*} \mathrm{~B}\left(\mathrm{~Hz}^{*} \mathrm{~T}\right)$ after 10 minutes heating.

These figures show that the temperature of the heater rises as $\mathrm{f} * \mathrm{~B}$ increases, and the heating value of the heater shows similarity when the condition that $\mathrm{f} * \mathrm{~B}$ is constant. Therefore, a higher heating can be obtained with lower magnetic flux density by increasing exciting frequency.

\subsection{Experiments in phantom}

The denaturing temperature of the egg white and brain are almost the same. So as a phantom model, egg white was used, whose shape was columnar and size

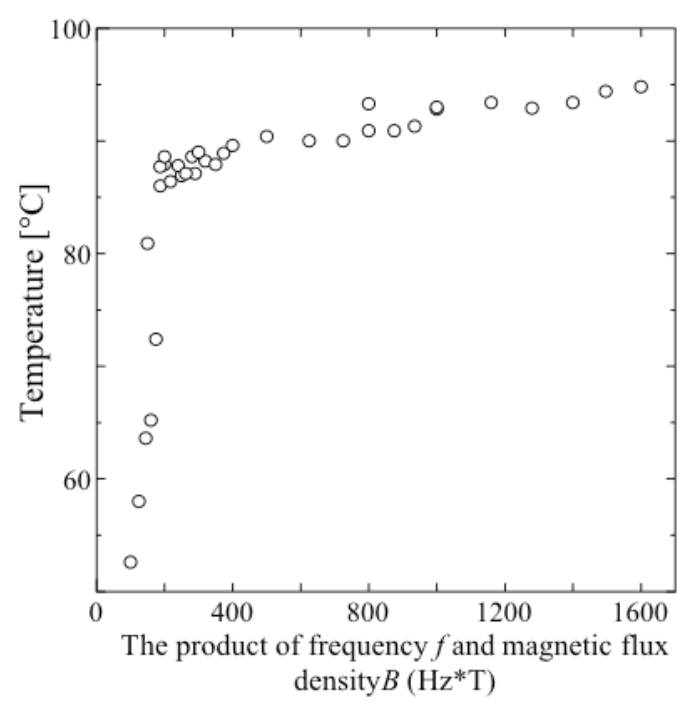

Fig. 13. The highest attainment temperature as a function of the product of Frequency $f$ and magnetic flux density $B$.

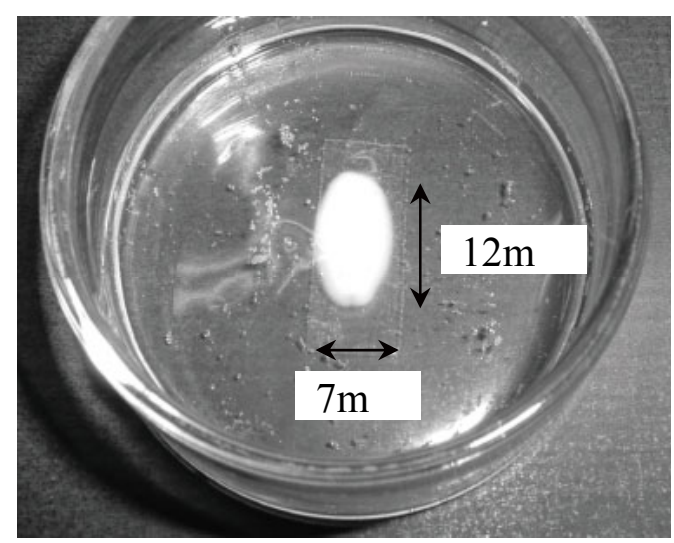

Fig. 14. The phantom after 10 minutes heating in the condition of $6 \mathrm{mT}-100 \mathrm{kHz}$.

was $40 \mathrm{~mm}$ in diameter and $10 \mathrm{~mm}$ in height. Figure 14 is the photograph of the phantom after 10 minutes heating in the condition of $6 \mathrm{mT}$ and $100 \mathrm{kHz}$. The temperature of the extent of $12 \mathrm{~mm}$ in length and $7 \mathrm{~mm}$ in width reached at $60{ }^{\circ} \mathrm{C}$ that the egg white was denatured. Based on these results, the greater area than Fig. 7 was heated to $60{ }^{\circ} \mathrm{C}$ in phantom when the condition that $\mathrm{f} * \mathrm{~B}$ was bigger than $600 \mathrm{~Hz}^{*} \mathrm{~T}$. It indicates that it is possible to heat the tumor locally.

\subsection{Conclusion}

We examined the effect of the exciting frequency on the heating value of a complex type of heater, using the "advanced" soft heating method as a heating process of hyperthermia. Consequently, it was found that higher heating can be obtained with lower magnetic flux density by increasing exciting frequency, and the 
heating value increases as the exciting frequency rises. The heated area in vitro was confirmed.

\section{Heat Element of Magnetic Hyperthermia in Mouse Melanoma Model 6.1. Purpose and outline}

We experimented in mouse melanoma model aiming at the application of the high temperature hyperthermia by which a soft heating method was applied to an actual mouse tumor. The purpose of this experiment is to confirm the effect of the high temperature hyperthermia in the tumor with the blood flow. In this research, authors experimented by using five mice' B16 melanoma (the tumor size: about $400 \mathrm{~mm}^{3}$ ).

\subsection{Experimental condition}

In the experiment, as a heat element the thermosensitive material is $\mathrm{Ni}-\mathrm{Cu}-\mathrm{Zn}$ ferrite $(0.6 \times 0.6$ $\times 10 \mathrm{~mm}$, permeability: 3500 , saturation magnetization: $263 \mathrm{mT}$ ), and curie-point is $70{ }^{\circ} \mathrm{C}$. If the Curiepoint is selected near $42.5^{\circ} \mathrm{C}$, which is the temperature of the tumor necrosis, the temperature of the tumor area tangent to the heater rises to $42.5{ }^{\circ} \mathrm{C}$, though the temperature of marginal area of the tumor does not rise to the point. So, it is necessary to use the higher Curiepoint than $42.5^{\circ} \mathrm{C}$ and attempt localized heating of the tumor. As a metal ring, the copper ring is selected $(0.05$ $\mathrm{mm}$ in thickness). Figure 15 is the picture of the heat element used in this experiment. The four elements are immersed under the mouse tumor to the square at about $5 \mathrm{~mm}$ intervals. The exciting condition is set to 200 $\mathrm{kHz}$ and $6 \mathrm{mT}$ and the plane type spiral coil is used as exciting instrument (Fig.16). And we used a fluorescent thermometer that has not affected by a high-frequency magnetic field.

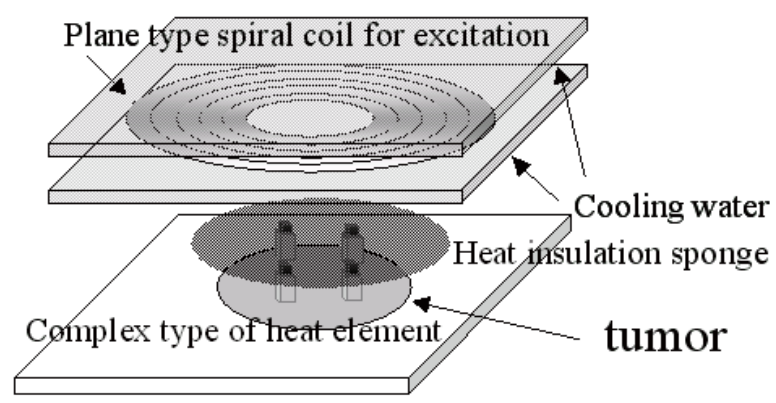

Fig. 16. Experimental environment.

\subsection{Temperature characteristic of the heat element}

Figure 18 shows the magnetic characteristic of the heat element as a function of time. The thermometer probe tip was located at the center of the metal ring.

It is the characteristic when the frequency is set to be constant, and the magnetic flux density range is $0.5 \mathrm{mT}$ - $2 \mathrm{mT}$. It can be said the higher magnetic flux density is, the more the characteristic of the heat element improves. Therefore, the exciting condition is set to 6 $\mathrm{mT}$ in test of actual tumor.

\subsection{Test of actual tumor}

Tumors of five mice are used for this experiment. Figure 19 shows temperature characteristic of marginal part of the tumor and rectum. The temperature of marginal part has reached $45{ }^{\circ} \mathrm{C}$ in all mice within ten minutes. It is shown that the entire tumor is raised to the necrosis temperature because the temperature of the heat element is raised to the Curie-point and in addition the marginal part has reached $45^{\circ} \mathrm{C}$. On the other hand, there is no influence to the temperature by calefactory of the tumor because the temperature of rectum that is the index of body temperature was not raised. The tumors are heated locally.

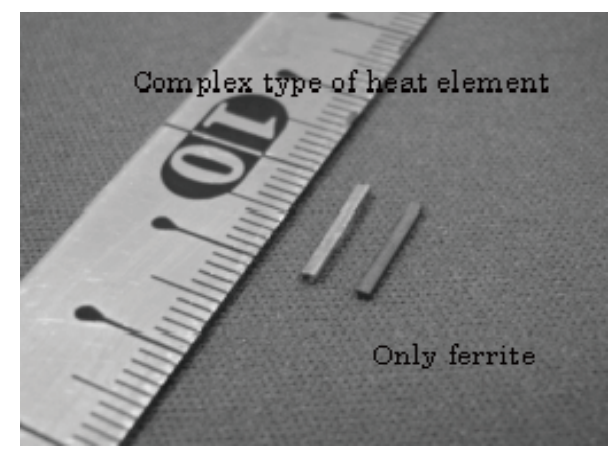

Fig. 15. Complex type of heat element.

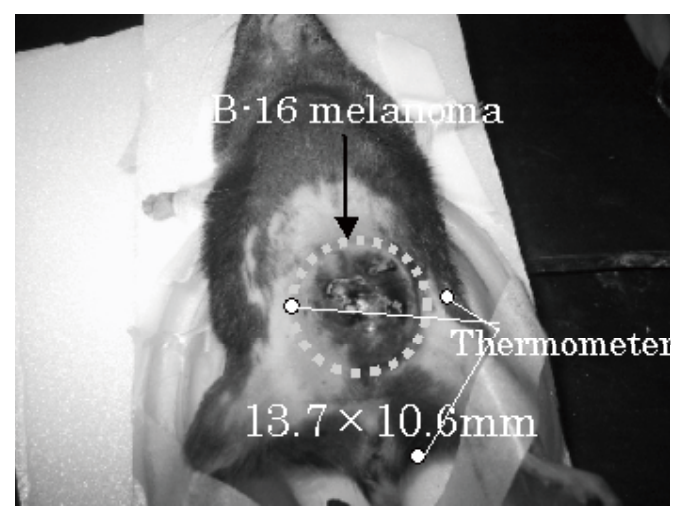

Fig. 17. Picture of B-16 melanoma. 


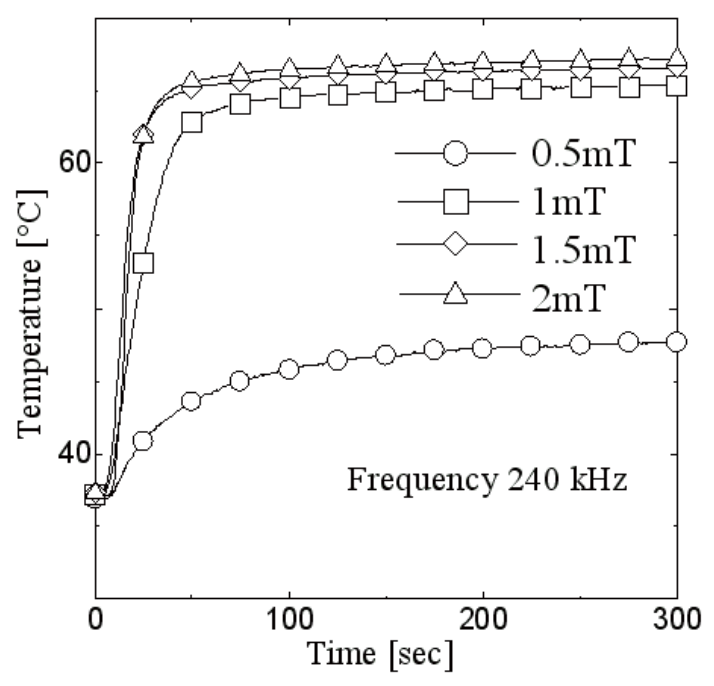

Fig. 18. Magnetic characteristic of the heat element.

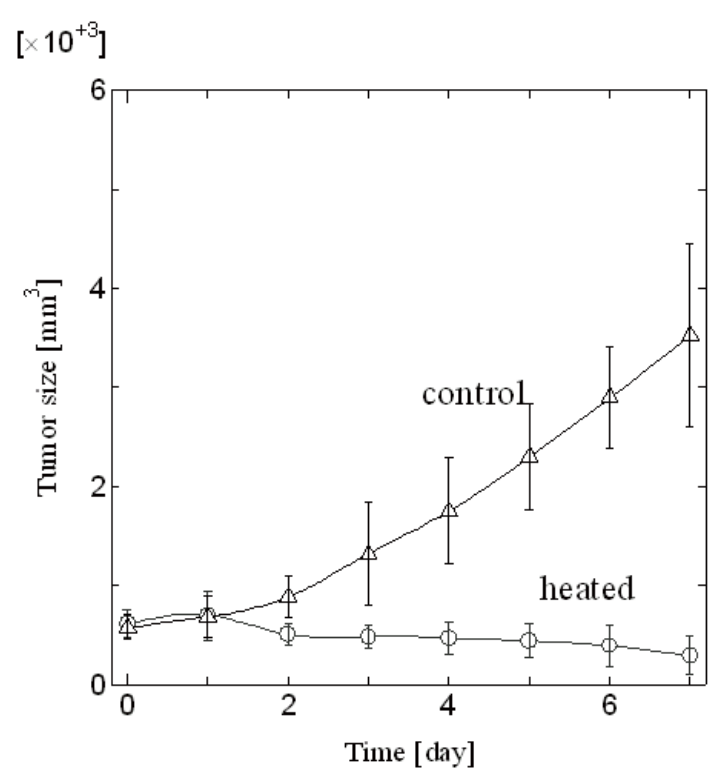

Fig. 20. The averaged tumor size of heated and control mice.

Figure 20 shows the difference of the averaged tumor size of heated and control mice. Heated line uses hyperthermia, and control line does not use hyperthermia. As a result, the proliferation of the tumor size is not seen in heated mouse though the size of the tumor in control mouse grows as time goes by. It is shown that the tumor of heated mouse is necrotized and the proliferation is not done. The tumor can be locally necrotized or remains small by the effect of the high temperature hyperthermia.

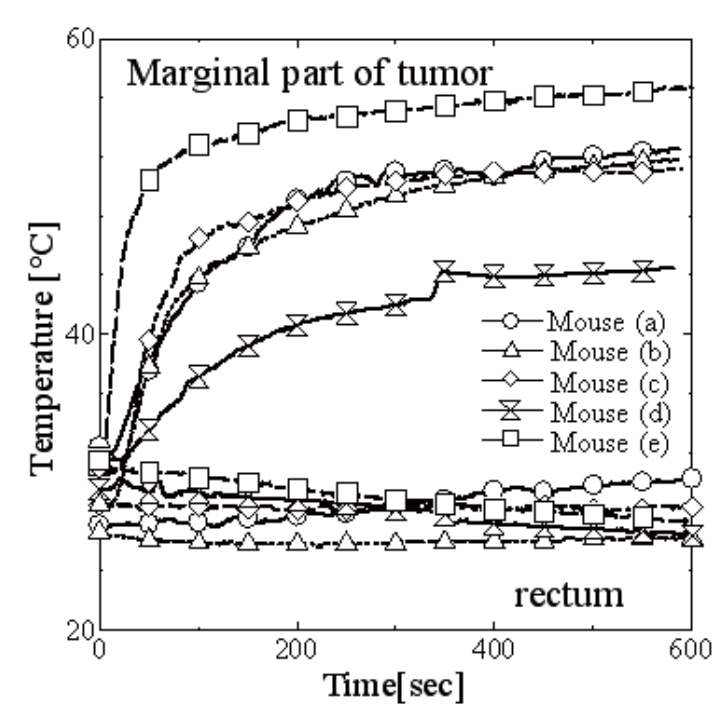

Fig. 19. Temperature characteristic of rectum and marginal part of tumor.
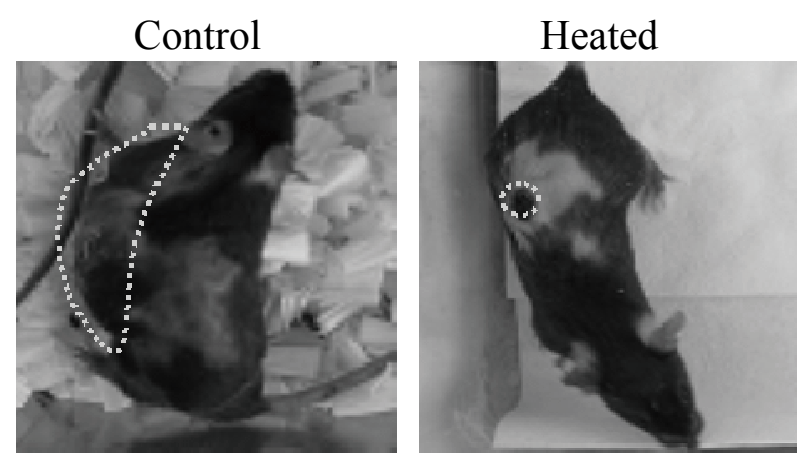

Fig. 21. Picture of the Mice 7 days later.

\subsection{Conclusion}

We examined whether the mice tumor in the blood flow can be necrotized locally through the four heat elements by the use of high temperature hyperthermia. As a result, in all mice the tumors are locally necrotized or remains small though in the blood flow. This is proven from not seeing the change in the tumor size even if time goes by. It is confirmed that the high temperature hyperthermia is effective to the necrosis of the cancer tumor.

Thinking about the application to the human body, the tumor has various shapes. It is necessary to arrange the elements for tumors of various shapes by combining elements with various Curie-point. 


\section{Conclusion}

This paper showed the progress of the functional hyperthermia system in our researches.

In the chapter 3, we examined temperature characteristics of heat elements with various volumes to adjust to the size of each tumor tissue. The results provided evidence that the temperature characteristics show a saturation tendency with an increase of the element volume. Based on this tendency, it seems reasonable to suggest that sufficient heating can be obtained, even on smaller element volumes.

In the chapter 4 , we examined the expansion of effective area. As a result, it is confirmed that the standard of $42.5^{\circ} \mathrm{C}$ or more effective area where tumor could be necrotized. It is assumed that no surgery such as insertion with injector etc., so it is essential to make the heater more small size and high performance. And we are now examining the transportation of the heater by magnetic micromachine that can drive in vivo.

In the chapter 5 , we examined the effect of the exciting frequency on the heating value of a complex type of heater. Consequently, it was found that higher heating can be obtained with lower magnetic flux density by increasing exciting frequency, and the heating value increases as the exciting frequency rises. The heated area in vitro was confirmed.

In the chapter 6 , we examined whether the mice tumor in the blood flow can be necrotized locally through the four heat elements by the use of high temperature hyperthermia. As a result, in all mice the tumors are locally necrotized or remains small though in the blood flow. This is proven from not seeing the change in the tumor size even if time goes by. It is confirmed that the high temperature hyperthermia is effective to the necrosis of the cancer tumor.

Thinking about the application to the human body, the tumor has various shapes. It is necessary to arrange the elements for tumors of various shapes by combining elements with various Curie-point.

\section{Acknowledgement}

Hidetoshi Matsuki acknowledges the support of Tohoku University Global COE Program "Global Nano-Biomedical Engineering Education and Research Network Centre".

\section{References}

[1] Matsuki H and Murakami K. High quality Soft heating method utilizing temperature dependence of permeability and core loss of low Curie temperature ferrite. IEEE Trans Magn MAG-21, 1927-1929, 1985.

[2] Murakami K and Matsuki H. Soft heating and its medical application. JARECT 21, 1986.

[3] Murakami K and Matsuki H. Thermosensitivity Magnetism Applied Engineering. Baifukan, Tokyo, Japan, 1-33, 135-140, 1993.

[4] Sato F, Jojo M, Matsuki H, Sato T, Sendoh M, Ishiyama K, and Arai KI. The Operation of Magnetic Micromachine for Hyperthermia and Its Exothermic Characteristic. IEEE Trans Magn 38, 3362-3364, 2002.

[5] Sato F, Suzuki N, Shimizu J, Matsuki H, and Sato $T$. Heat Characteristics of Micro Magnetic Heat Elements for Advanced Hyperthermia. IEEE Trans Magn 40, 2967-2969, 2004.

[6] Shimizu J, Sato F, Matsuki H, Sato T, Sendou M, Ishiyama K, and Arai K. Effective Area of Hyperthermia Using Implanted Heaters. J Magn Soc Jpn 27, 456-459, 2003.

[7] Sendoh M, Ishiyama K, Arai KI, Jojo M, Sato F, and Matsuki H. Fabrication of Magnetic Micromachine for Local Hyperthermia. IEEE Trans Magn 38, 33593361, 2002.

[8] Sawaya Y, Suzuki N, Sato F, Matsuki H, Aiba S, and Sato T. Examination of the exciting condition for the high-temperature magnetic hyperthermia. IEEE Trans Magn 41, 4161-4163, 2005.

[9] Matsuki H, Sato F, Sawaya Y, Maruyama T, Aiba S, Ito Y, and Sato T. Mechanism of Self Control Type Hyperthermia Bassed on Advanced Soft Heating. Jpn J Hyperthermic Oncol 22, 2006. 